Ergod. Th. \& Dynam. Sys. (1982), 2, 543-544

Printed in Great Britain

\title{
Index to Volume 2
}

ALEXEYEV, V. M. Existence of a bounded function of the maximal spectral type, 259

ALEXEYEV, V. M. AND OSIPOV, YU. S. Accuracy of Kepler approximation for fly-by orbits near an attracting centre, 263

ARNOL'D, V. I. Singularities of Legendre varieties, of evolvents and of fronts at an obstacle, 301

BALLMANN, W. AND BRIN, M. On the ergodicity of geodesic flows, 311

BLOCK, L. AND HART, D. The bifurcation of homoclinic orbits of maps of the interval, 131

BI.OCK, L. AND HART, D. The bifurcation of periodic orbits of one-dimensional maps, 125

Brin, M. See BallmanN, W. and Brin, M.

DANI, S. G. On uniformly distributed orbits of certain horocycle flows, 139

ElLis, R. AND Glasner, S. Iterated extensions, 1

FILIPKIEWICZ, R. P. Isomorphisms between diffeomorphism groups, 159

FRIED, D. Isentropic fitting of Anosov automorphisms, 173

Glasner, S. See Ellis, R. And Glasner, S.

HaRt, D. See Block, L. AND Hart, D.

HofBAUER, F. AND KELlER, G. Equilibrium states for piecewise monotonic transformations, 23

JACOBSON, M. V. Invariant measures for some one-dimensional attractors, 317

KATOK, A. Entropy and closed geodesics, 339

KATOK, A. Some remarks on Birkhoff and Mather twist map theorems, 185

Keller, G. See Hofbauer, F. ANd Keller, G.

KIFER, Y. AND SLUD, E. Perturbations of random matrix products in a reducible case, 367

KRIEGER, W. On the subsystems of topological Markov chains, 195

Ledrappier, F. AND STRElcyn, J.-M. A proof of the estimation from below in Pesin's entropy formula, 203

LEHRER, E. AND WEISS, B. An $\varepsilon$-free Rohlin lemma, 45

L.IND, D. A. Dynamical properties of quasihyperbolic toral automorphisms, 49

MARGULIS, G. A. Finitely-additive invariant measures on Euclidean spaces, 383

MATHER, J. N. Glancing billiards, 397.

MisiurewiCZ, M. Attracting Cantor set of positive measure for a $C^{\infty}$ map of an interval, 405

MisiurewiCZ, M. Periodic points of maps of degree one of a circle, 221

MisiurewiCZ, M. Perturbations and transitivity for certain maps of an interval, 229

MOECKEL, R. Geodesics on modular surfaces and continued fractions, 69

Moussu, R. Symétrie et forme normale des centres et foyers dégénérés, 241

Osıpov, Yu. S. See AlexeyeV, V. M. AND Osipov, Yu. S.

PEsin, YA. B. AND SinAI, YA. G. Gibbs measures for partially hyperbolic attractors, 417

PrZYTYCK1, F. Examples of conservative diffeomorphisms of the two-dimensional torus with coexistence of elliptic and stochastic behaviour, 439 
RuDOLPH, D. J. A mixing Markov chain with exponentially decaying return times is finitarily Bernoulli, 85

RuDOLPH, D. J. Ergodic behaviour of Sullivan's geometric measure on a geometrically finite hyperbolic manifold, 491

RuELLE, D. Repellers for real analytic maps, 99

SARNAK, P. Entropy estimates for geodesic flows, 513

Sinai, Ya. G. See Pesin, Ya. B. And Sinal, Ya. G.

Slud, E. See Kifer, Y. AND Slud, E.

STRElcyn, J.-M. See LedRAPPIER, F. AND STRElCyN, J.-M.

WoJTkowski, M. On the ergodic properties of piecewise linear perturbations of the twist map, 525

YounG, L.-S. Dimension, entropy and Lyapunov exponents, 109 